\title{
Guiding Lead Optimization with GPCR Structure Modeling and Molecular Dynamics
}

Alexander Heifetz ${ }^{1 *}$, Tim James ${ }^{1}$, Inaki Morao ${ }^{1}$, Michael J. Bodkin ${ }^{1}$ and Philip C. $\operatorname{Biggin}^{2 *}$

${ }^{1}$ Evotec Ltd, 114 Innovation Drive, Milton Park, Abingdon, Oxfordshire, OX14 4RZ, UK ${ }^{2}$ Department of Biochemistry, University of Oxford, South Parks Road, Oxford, OX1 3QU.

*Corresponding author. Tel.: +44 (0)1235 8389 25; Fax: +44 (0)1235 863139

E-Mail address: Alexander.Heifetz@Evotec.com and philip.biggin@bioch.ox.ac.uk

Keywords: Structure based drug design; molecular dynamics; simulation; ion channels; glutamate receptor; G-protein coupled receptor; docking; virtual screening. 


\section{Abstract}

GPCR modeling approaches are widely used in the hit-to-lead (H2L) and lead optimization (LO) stages of drug discovery. Modern protocols that involve molecular dynamics (MD) simulation can address key issues such as the free energy of binding (affinity), ligand-induced GPCR flexibility, ligand binding kinetics, conserved water positions and their role in ligand binding and the effects of mutations. The goals of these calculations are to predict the structures of the complexes between existing ligands and their receptors, to understand the key interactions and to utilize these insights in the design of new molecules with improved binding, selectivity or other pharmacological properties. In this review we present a brief survey of various computational approaches illustrated through a hierarchical GPCR modeling protocol (HGMP) and its prospective application in three drug discovery projects. We also describe the fragment molecular orbital $(F M O)$ quantum mechanical $(\mathrm{QM})$ method for the detailed exploration of receptor-ligand interactions. 


\section{Introduction}

G-protein coupled receptors (GPCRs) are a particularly well-studied family of membrane proteins. Not only are they a large and important group of signaling proteins, they are also the targets for about $40 \%$ of all therapeutic compounds [1] in clinical use [2-4]. Although over 800 human proteins are classified as GPCRs [5], drugs have only been developed against $<10 \%$ of these. Hence, there is huge potential for new GPCR-targeted therapeutics [6].

Recent advances in X-ray crystallography have shed light on the structure, function and pharmacology of GPCRs and thus facilitated structure-based drug discovery (SBDD) $[1,7]$. However, GPCR crystallography is not currently feasible for every receptor or receptor-ligand complex and that significantly limits the ability of this method to guide drug discovery for GPCR targets in "real-time". Furthermore, experimentally determined structures represent snapshots of what we know are very dynamic entities and as a consequence offer only limited insights into the overall conformational space and related functions [8].

In the absence of crystallographic data, GPCR modeling is a practical alternative that is widely used to guide SBDD $[9,10]$. Modern computational approaches can address key issues such as GPCR flexibility and ligand-induced dynamics, ligand kinetics $\left(\mathrm{k}_{\mathrm{o}} / \mathrm{k}_{\mathrm{off}}\right.$ rates $)$, prediction of water positions and their role in ligand binding and prediction of the effects of mutations on ligand binding. However, the general goals of every modeling protocol are 1) to predict the structures of the complexes between existing ligands and a target receptor, 2) to explore the key interaction between these ligands, surrounding residues and water molecules and 3) to utilize these insights in the design of new molecules with improved binding, selectivity or other pharmacological properties.

The lead optimization phase of drug discovery is usually defined as the process of bringing a chemical series to clinical trials through iterative steps of design and testing [11]. Compared to H2L, the initial lead compound(s) in LO have typically already demonstrated significant potency against the 
target. However, the affinity, selectivity or other pharmacological properties might need further optimization. The improvement of what are often already potent compounds can be challenging and requires detailed information on the interactions between the ligand and its target and off-target receptors. Any modeling input must therefore be accurate and give reliable insights at the molecular level.

\section{GPCR modeling protocols}

In spite of the rapid growth in the number of GPCR-ligand structures being experimentally solved [5], their direct impact on LO remains limited. The low throughput of experimental methods means that structures relevant to the specific chemotype of interest are rarely available in a timely manner. Therefore, if one wants to pursue any kind of structure-based approach, modeling is commonly the only viable solution [12]. Even in cases where the sequence identity of the available templates is low, careful model building in conjunction with site-directed mutagenesis and binding assays can be very useful in rationalizing existing SAR data and determining the future direction of a drug discovery program [13].

Traditional GPCR homology modeling approaches [10] often involve the following steps: (1) sequence alignment between the modeled receptor and an appropriate template, (2) homology modeling and refinement and (3) docking of ligands into the binding site. The key problem of such 'static' approaches is that the modeled receptor is practically a 'twin' of the template and therefore some of the more subtle structural features are often missed. This significantly reduces the relevance of the models and their ability to guide SBDD, which is problematic in the LO stage of drug discovery when information on the fine details of the system is particularly important. Furthermore, most of standard docking protocols keep the receptor (largely) rigid, and so do not address the issue of receptor flexibility. As these protocols do not take into account the ligand-induced (or ligand-stabilized) conformation of the receptor, it makes it harder to rationalize the effects of ligands in terms of agonism or antagonism. Some docking approaches assign limited flexibility to the sidechains of key residues. 
However, this approach is too artificial and is an unsatisfactory solution to the general problem of receptor flexibility. The position and role of water-mediated interactions has also been largely ignored, although recently there have been efforts to address this.

Modern GPCR modeling protocols $[14,15]$ have moved beyond the use of static homology modeling approaches by performing the type of extensive refinement and exploration of both structure and flexibility that is required to drive LO [16]. To address the various challenges of GPCR drug discovery programs [17], these contemporary approaches are encapsulated as toolkits that can be flexibly assembled into workflows tailored to the specific needs of each project. The ability to incorporate experimental data during the modeling is another important factor that can enhance the effectiveness of these workflows.

An example of such a workflow is the hierarchical GPCR modeling protocol (Figure 1) [13,16]. The HGMP generates a GPCR model and its potential complexes with small molecules by applying a series of computational methods. The protocol makes use of homology modeling followed by MD simulations and docking (flexible docking if required) to predict binding poses and functions of ligands. The HGMP is a toolbox for GPCR modeling where experimental data can be easily fed in. It is equipped with GPCR-specific "plugins”, including a GPCR-likeness assessment score (GLAS) to evaluate model quality [18] and a pairwise protein comparison method (ProS) used to cluster structural data and distinguish between different activation substates [18]. The HGMP has been applied in a number of industrial drug design projects, which have also led to further refinements of the protocol. 


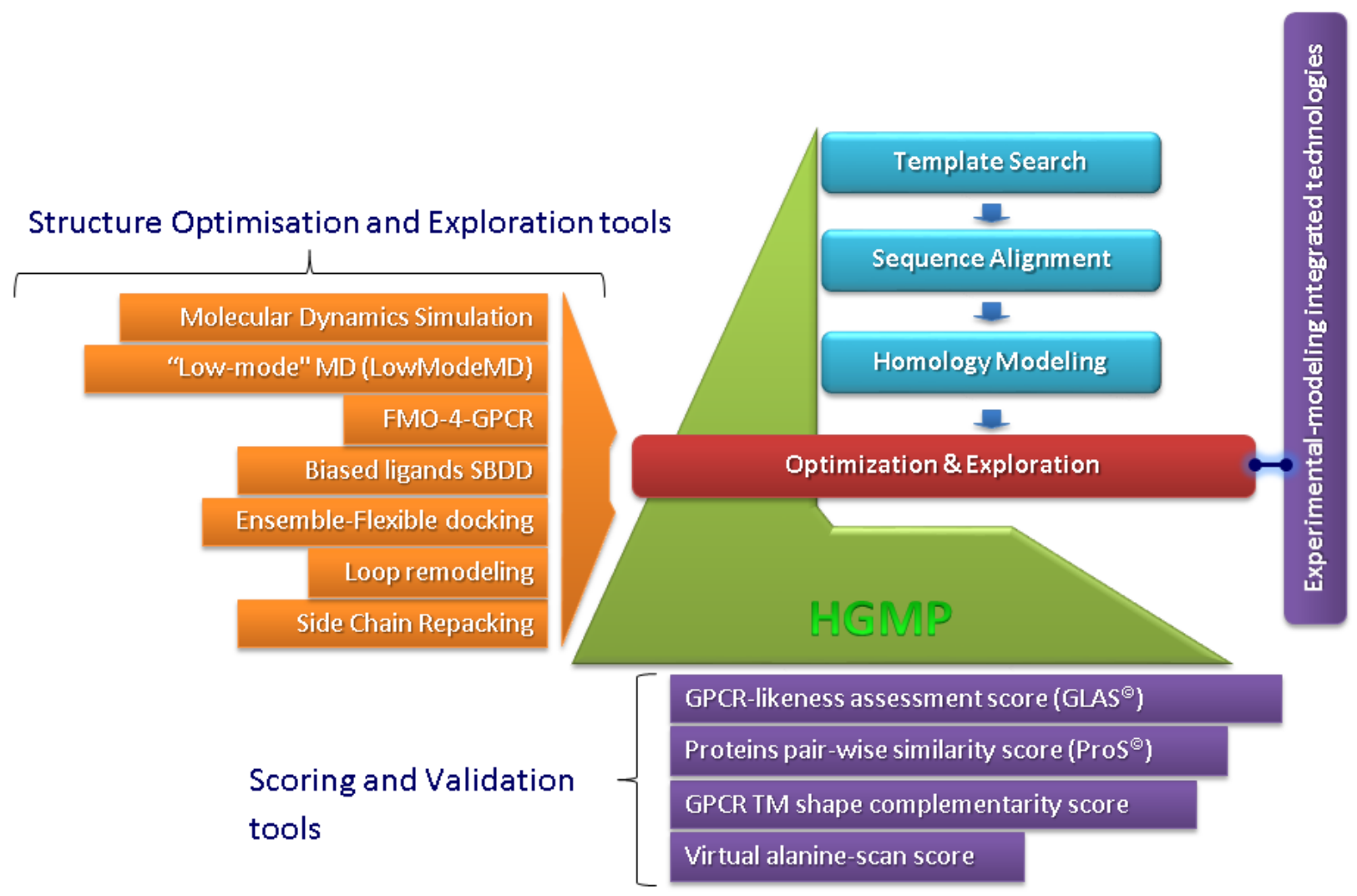

Figure 1: A summary schematic of the Hierarchical GPCR Modeling Protocol (HGMP)

\section{MD simulation as a tool for GPCR SBDD}

GPCRs are, by functional necessity, very dynamic entities. MD simulation therefore provides an invaluable source of structural and functional information for these proteins. It can be used in a variety of ways including refinement of the homology model in a more realistic membrane environment, exploration of ligand-induced flexibility and function, the analysis of solvent, the effect of mutation on receptor stability and exploration of ligand binding kinetics [14]. MD trajectories are often used to generate an ensemble of possible receptor substates [18]. The ProS and GLAS methods outlined about were developed to explore the structural data generated within MD simulations and to help distinguish between different GPCR substates [18]. 
Another area that has received increasing attention over recent years has been the role of water networks and their elucidation by MD simulation [14]. With the availability of high resolution GPCR structures (e.g., the $\delta-O R[19]$ or the $A_{2 A} R[20]$ with resolutions of $1.8 \AA$ ) it has become clear that GPCRs often exhibit conserved water networks and that these play an important role in receptor signaling [21] and ligand binding. In order for a ligand to displace a water molecule, there has to be an overall benefit in terms of free energy. Several alternative methods like WaterMap [1], WaterFLAP [1], WaterDock [22] and 3D-RISM [23] are available that enable a relatively quick estimation of the energy penalty for water displacement [24]. They can help medicinal chemists to decide whether to interact with or displace a certain water molecule, if a particular sub-pocket of the receptor can be explored by hydrophobic moieties or if a displaced water has to be substituted by a group that mimics the hydrogen bond network. These quick methods provide only an estimate of the free energy, but often they are good enough for a go/no-go decision and are intensively used in LO programs [1].

In recent years there has been growing interest in trying to relate structure to kinetics [25]. The kinetics of drug-binding [26] are increasingly being recognized as important for clinical effectiveness [27]. Indeed, it has been shown experimentally that there is a positive correlation between functional efficacy and "residence time" (RT) at the receptor [28,29]. There is substantial evidence $[20,28,30,31]$ that $\sim 70 \%$ of long RT therapeutics display higher efficacy than comparable faster-dissociating drugs [28], supporting a growing recognition that drug-target RT may be of even greater therapeutic importance than affinity. Several recent reviews have emphasized the pivotal role of RT optimization in the LO phase of drug discovery [28,30], suggesting that detailed structure-based studies of RT should be introduced in the earlier phases of drug discovery to prevent "fail late, fail expensive" scenarios. It has been recently demonstrated that MD simulation can be a promising tool for the exploration and structural rationalization of ligand RT [32].

MD simulations also allow one to explore the possibility of allosteric and cryptic binding pockets [33]. The latter are not exposed to bulk solvent all of the time and so may be hidden in certain 
crystallographic structures. MD allows these sites to manifest themselves [34] and so permit docking and similar protocols to be followed in the usual manner. Simulations are also essential for understanding the mechanisms of allosteric modulation. In some cases though, full MD simulation may not be required when, for example, just local refinement of a homology model is required. In such cases "low-mode" molecular dynamics [35] (LowModeMD) simulation can provide a quicker solution.

\section{Case study 1: Discovery of selective 5-HT $2 c$ agonists for the treatment of metabolic disorders}

In this project [36], which was performed prior to the crystal structures of $5-\mathrm{HT}_{2 \mathrm{~B}}$ and $5-\mathrm{HT}_{1 \mathrm{~B}}$ being published, the challenge was to find novel $5-\mathrm{HT}_{2 \mathrm{c}}$ agonists that that did not also activate $5-\mathrm{HT}_{2 \mathrm{~A}}$ and $5-\mathrm{HT}_{2 \mathrm{~B}}$. The HGMP was applied to model both the active and inactive receptor conformations, referred to as $5-\mathrm{HT}_{2 \mathrm{C}}$ active and $5-\mathrm{HT}_{2 \mathrm{C}}$ inactive, respectively. Models were also built of the off targets, 5$\mathrm{HT}_{2 \mathrm{~A}}$ and 5-HT $\mathrm{T}_{2 \mathrm{~B}}$. Flexible docking was then applied to predict the binding modes of compounds in 5$\mathrm{HT}_{2 \mathrm{~A}}, 5-\mathrm{HT}_{2 \mathrm{~B}}$ and 5- $\mathrm{HT}_{2 \mathrm{C}}$.

The binding site of $5-\mathrm{HT}_{2 \mathrm{C}}$ inactive was proposed to be shallower than that of $5-\mathrm{HT}_{2 \mathrm{C}}$ active due to residues from TM3 and TM6 forming stabilizing inter-helical interactions in the former [16]. It was hypothesised that these inter-helical interactions are broken in the active conformation of the $5-\mathrm{HT}_{2 \mathrm{C}}$ receptor, which is stabilized by agonist molecules entering deeper into the binding site and compensating via interactions with various other residues. These include $W 324^{6.48}$, a key residue previously identified as a "transmission switch" residue [37-39] and which may form part of a larger "hydrophobic hindering mechanism" [40]. Agonists were proposed to interact simultaneously with both TM3 and TM6 in 5- $\mathrm{HT}_{2}$ active, thus increasing the overall stability of $5-\mathrm{HT}_{2 \mathrm{C}}$ active and promoting activation. Furthermore, these modeling observations (which were directly supported by the published SDM data) were incorporated into the design of novel 5- $\mathrm{HT}_{2 \mathrm{C}}$ agonists [36]. Hits were also assessed for hERG liability via docking to a model of the hERG channel. The result was the discovery of a novel

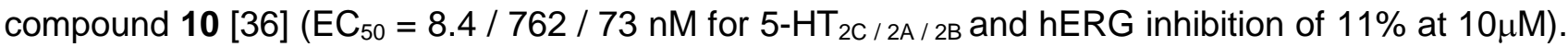
The whole design cycle for this project is summarized in Figure 2. 


\section{Primary lead \& new ideas from chemistry team}
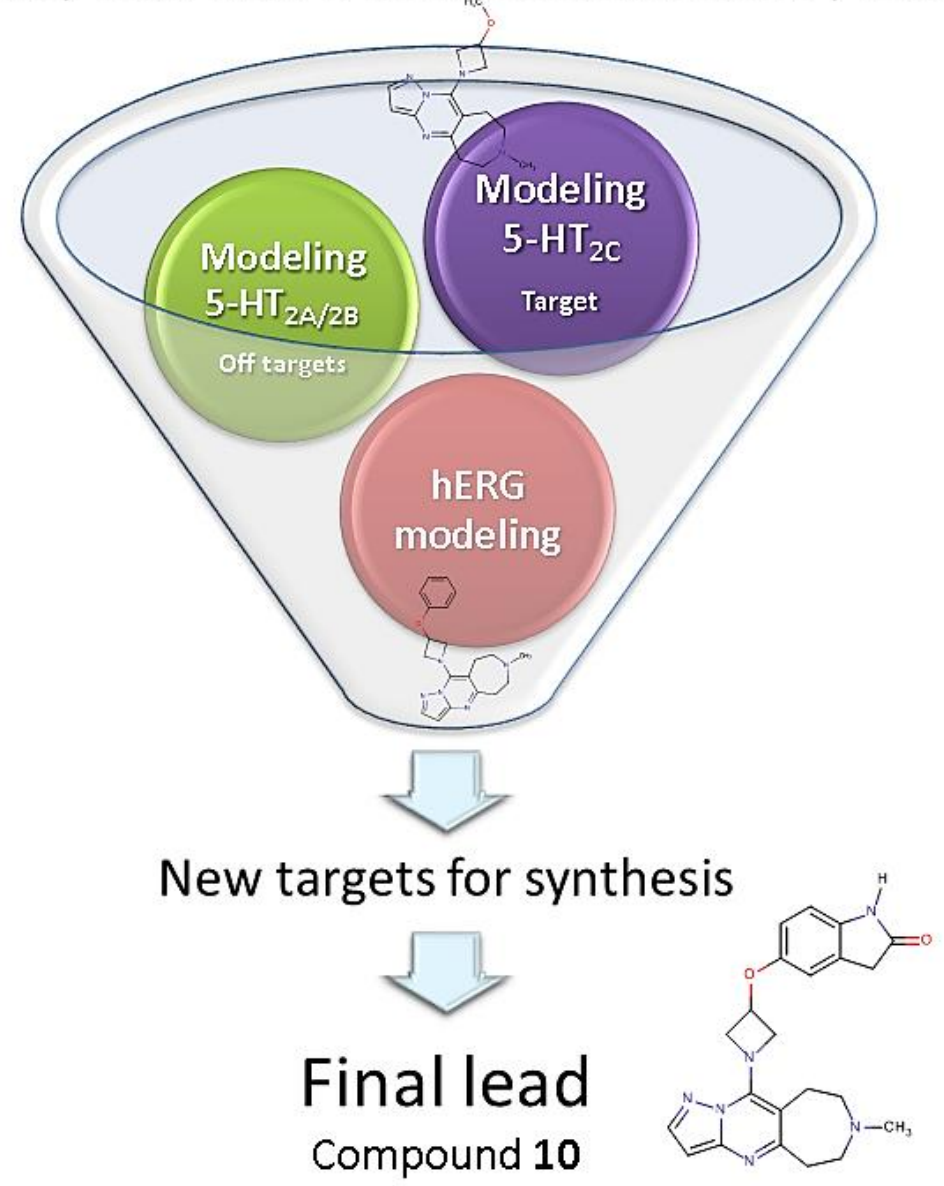

Figure 2: Design cycle/filter for potent, selective $5 \mathrm{HT}_{2 \mathrm{C}}$ antagonists lacking hERG activity [36].

\section{Case study 2: Fighting obesity with a sugar-based library}

Obesity is an increasingly common condition. Although antagonism of the melaninconcentrating hormone-1 receptor $(\mathrm{MCH}-1 \mathrm{R})$ has been widely reported as a promising therapeutic avenue for obesity treatment, no $\mathrm{MCH}-1 \mathrm{R}$ antagonists have reached the market. Discovery and optimization of new compounds targeting $\mathrm{MCH}-1 \mathrm{R}$ has been hindered by low high throughput screening (HTS) success rates and a lack of structural information about the $\mathrm{MCH}-1 \mathrm{R}$ binding site. In this project, a novel approach that integrated GPCR modeling with design, synthesis and screening 
of a diverse library of sugar-based compounds from the VAST technology (Versatile Assembly on Stable Templates) was used, to provide structural insights on the $\mathrm{MCH}-1 \mathrm{R}$ binding site [41].

The 490 VAST compounds obtained from this library design were screened against MCH-1R, resulting in the discovery of a moderately potent $\mathrm{MCH}-1 \mathrm{R}$ antagonist, ACL21823 (radioligand binding to $\mathrm{MCH}-1 \mathrm{R}$ gave an $\mathrm{IC}_{50}=306 \mathrm{nM}$, see Figure 3). The discovery of ACL21823 was utilized in the construction of a high quality $\mathrm{MCH}-1 \mathrm{R}$ model and the refinement of its antagonist binding site [41]. The quality of the $\mathrm{MCH}-1 \mathrm{R}$ model was validated by a virtual enrichment experiment and the modeldriven structure-based expansion of ACL21823, which allowed the generation of a list of key $\mathrm{MCH}$ $1 \mathrm{R}$ residues potentially involved in antagonist binding. The GPCR-VAST method demonstrates how ligand SAR data, when combined with modeling, can provide a useful source of structural information on GPCR binding sites [41]. The usefulness of this method to drug discovery was demonstrated by a structure-based virtual screen, which achieved a hit rate of $14 \%$ and yielded 10 new chemotypes of $\mathrm{MCH}-1 \mathrm{R}$ antagonists including EOAI3367472 $\left(\mathrm{IC}_{50}=131 \mathrm{nM}\right)$ and EOAI3367474 $\left(\mathrm{IC}_{50}=213 \mathrm{nM}\right)$. 


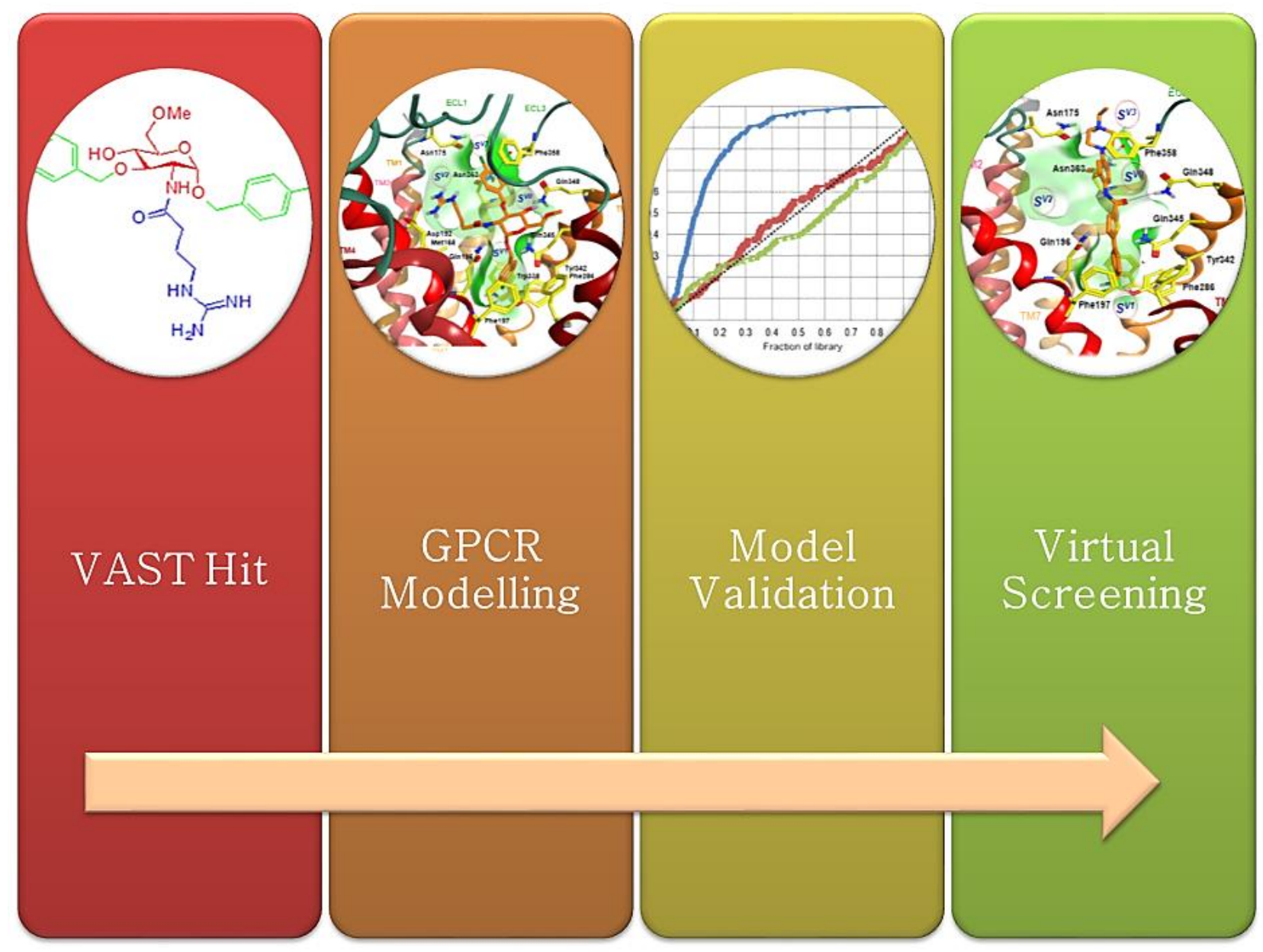

Figure 3: Summary schematic of the VAST-GPCR modeling workflow that led to the discovery of new MCH$1 \mathrm{R}$ antagonists [41].

\section{Case study 3: Discovery of potent \& selective $\mathrm{OX}_{2}$ receptor antagonists}

The orexin receptors $\left(O X_{1}\right.$ and $\left.O X_{2}\right)$ are linked to a range of different physiological functions including the control of feeding, energy metabolism, modulation of neuro-endocrine function and regulation of the sleep-wake cycle [42]. The key challenges of this project were to increase the $\mathrm{OX}_{2}$ activity and selectivity over $\mathrm{OX}_{1}$. This was particularly difficult as $\mathrm{OX} \mathrm{X}_{1}$ and $\mathrm{OX}_{2}$ receptors have over $80 \%$ sequence similarity. This project was completed before the crystal structures of $\mathrm{OX}_{1}$ and $\mathrm{OX}_{2}$ bound to Suvorexant were solved [43]. We used molecular dynamics simulation to study this selectivity question [44]. The simulations suggested that differences in intra-helical interactions resulted in differences in TM conformation and differences in topology of the binding pocket. The 
differences were small but sufficient to design molecules with $\mathrm{OX}_{2}$ selectivity. This rational design significantly decreased the amount of synthesis required by focusing the effort on the relevant portion of the ligand structure, as outlined in Figure 4. The final compound, EP-009-0513, had $K_{i}$ values of 4,363 and $5.7 \mathrm{nM}$ for $\mathrm{OX}_{1}$ and $\mathrm{OX}_{2}$, respectively.

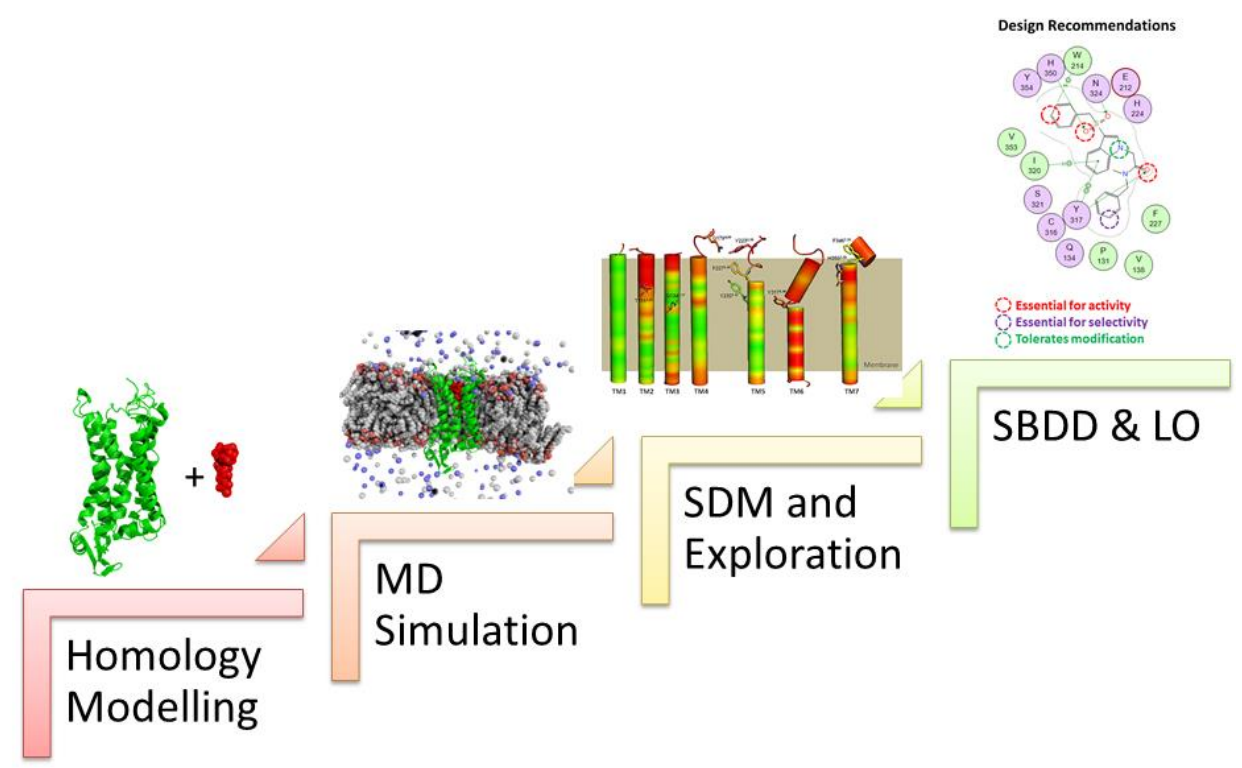

Figure 4: Schematic summarizing how interaction maps derived from structural models and MD data can be used to provide synthesis recommendations [18].

\section{Exploring Receptor-Ligand Interactions}

The understanding of binding interactions between a protein and a small molecule plays a key role in the rationalization of potency and selectivity [45]. However, even with the crystal structure in hand, visual inspection and force-field based molecular mechanics calculations cannot always explain the full complexity of the molecular interactions that are so critical in LO. Quantum mechanical methods have the potential to address this shortcoming, but the high computational cost has typically made the use of these calculations impractical.

The Fragment Molecular Orbital (FMO) method is widely used by us $[45,46]$ in protein-ligand binding calculations and drug design because it offers substantial computational savings over 
traditional QM methods (Figure 5a). By dividing the system, both ligand and receptor, into smaller pieces and performing QM calculations on these fragments, one can achieve high efficiency. A typical FMO calculation on a GPCR-ligand complex takes approximately $4 \mathrm{~h}$ on $36 \mathrm{CPU}$ cores to complete, significantly faster than equivalent classical QM calculations.

Using FMO, one can take a protein-ligand complex and calculate a list of interactions and their chemical natures. Many of these interactions are difficult to detect or quantify with non-QM methods. An example is shown in Figure $5 b$ for the binding of $\mathrm{OX}_{2}$ to its antagonist Suvorexant [45]. This information is very useful in guiding rational LO in terms of ligand modifications such as scaffold replacement and linking or the extension of chemical moieties to form stronger or new interactions with the protein. Alternatively, it can also be applied to the discovery of the new ligands.

(a)

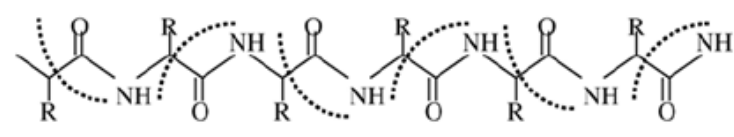

Fragmentation of peptide chain

$$
\Delta E_{i j}^{\mathrm{int}}=\Delta E_{i j}^{\mathrm{ES}}+\Delta E_{i j}^{\mathrm{CT}}+\Delta E_{i j}^{\mathrm{DI}}+\Delta E_{i j}^{\mathrm{EX}}
$$

PIE (Pair Interaction Energy) between fragments $i$ and $j$

Electrostatic $\left(\Delta E^{E S}\right)$

Forces between point charges, permanent and induced.
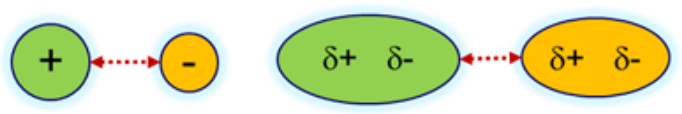

Charge transfer $\left(\Delta E^{C T}\right)$

Interactions between occupied orbitals of a donor and unoccupied orbitals of an acceptor. Orbital energy gap and overlap are the important factors.

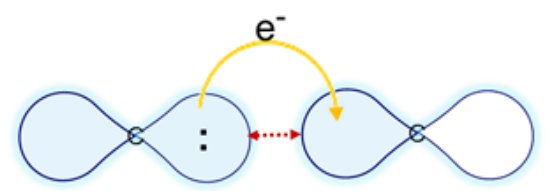

Dispersion $\left(\Delta E^{D I}\right)$

Interaction forces due to instantaneous polarization multipoles caused by the movement of electrons in nearby molecules

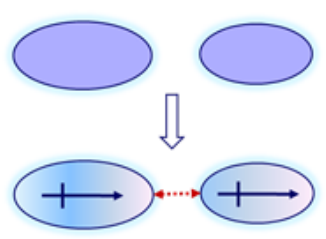

\section{Exchange repulsion $\left(\Delta E^{E X}\right)$}

Repulsive forces between molecules that are close together, mainly due to the overlap of occupied orbitals

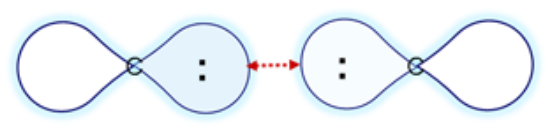


(b)

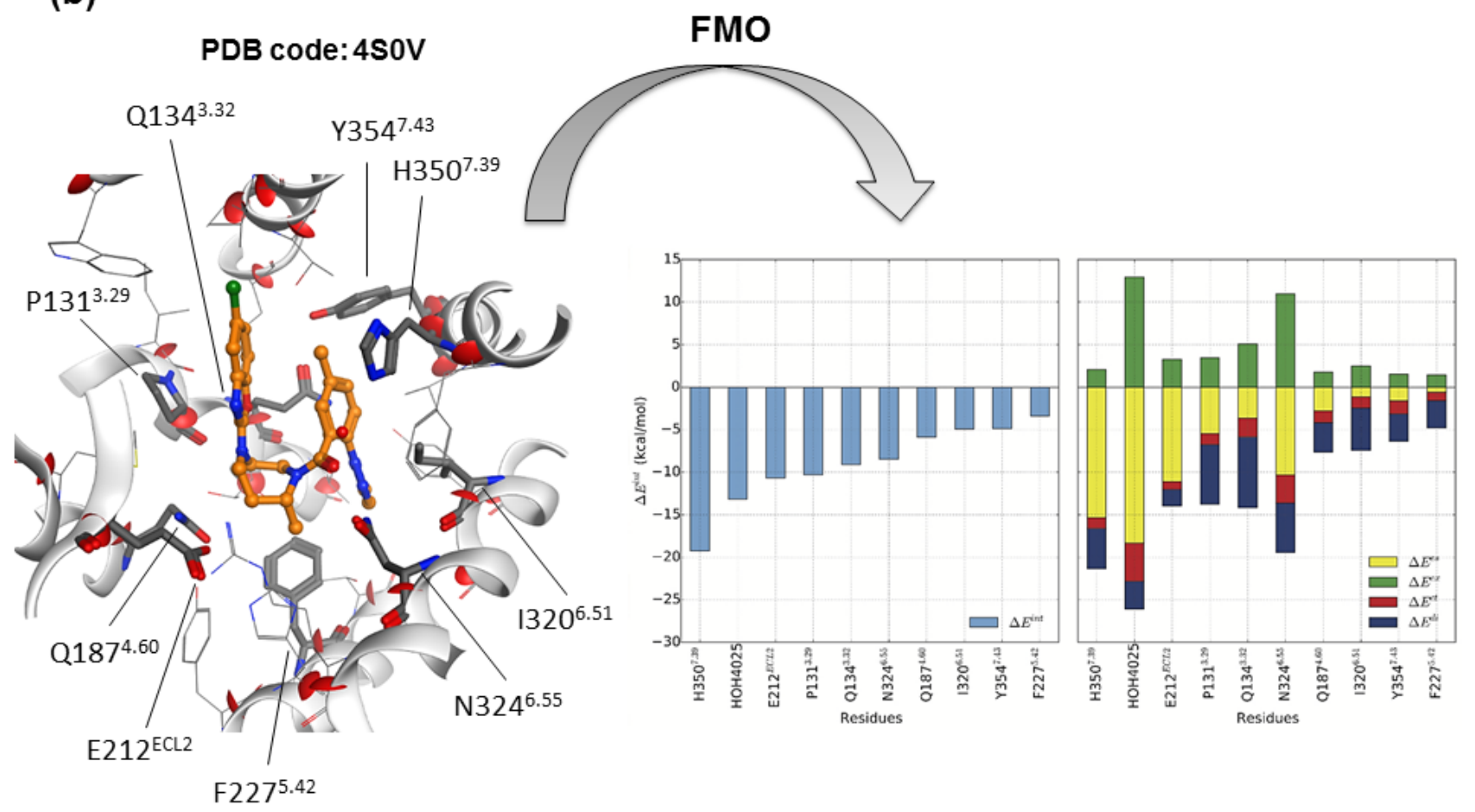

Figure 5: Schematic summary of the FMO approach: (a) Workflow for PIEDA calculations and details on each of the PIE terms that are computed (b) FMO analysis of human adenosine $\mathrm{OX}_{2}$ receptor in complex with Suvorexant (PDB ID 4S0V [47]). The carbon atoms of the ligand are shown in light orange and for the receptor are grey. Nitrogen atoms are shown in blue, oxygen in red and chlorine in light green. The fragmented bonds are marked as red discs. The left-hand bar plots describe the sorted PIE of the most significant residues, and the right-hand plots describe the pair interaction energy decomposition analysis (PIEDA) of these key interactions. PIE terms: electrostatics, dispersion, charge-transfer, and exchange-repulsion are color-coded in yellow, blue, red, and green, respectively. The figure is adapted from our previous publication [45].

\section{Conclusion}

Modern GPCR modeling protocols, such as the HGMP, have gone beyond the use of static models and allow for the type of detailed exploration of GPCR-ligand structures required to drive LO. In this review, we have illustrated how these methods, in combination with experimental data, can account for protein flexibility to rationalize not only binding affinity but also selectivity of GPCR agonists and antagonists. These methods allow the prediction of GPCR substates in a way that is not possible with static homology modeling alone. 


\section{Future challenges}

In spite of huge efforts in the pharmaceutical industry to design novel drugs for GPCR targets there remains tremendous attrition along R\&D pipelines [28]. Many drug candidates eventually fail in clinical trials due to a lack of efficacy $[28,30]$. A retrospective analysis of successfully launched drugs revealed that their beneficial effects in patients may be attributed to their long drug-target residence times. Over the past decade, the kinetics of ligand binding has received increasing interest and the concept of RT is recognized as an invaluable parameter for early drug optimization. In spite of the importance of the RT, there is no clear method to optimize this parameter. Currently the in-silico prediction of association/dissociation rates is only possible for very small fragments rather than druglike molecules. Therefore the state-of-the-art is still the explanation of experimental off-rates of druglike molecules rather than their prediction. However, this will no doubt change as researchers explore this area further.

\section{Conflict of interest statement}

Nothing declared.

\section{References and recommended reading}

Papers of particular interest, published within the period of review, have been highlighted as:

- $\quad$ of special interest

- $\quad$ of outstanding interest

1. Cooke RM, Brown AJ, Marshall FH, Mason JS: Structures of G protein-coupled receptors reveal new opportunities for drug discovery. Drug Discov Today 2015, 20:1355-1364.

- Important review that describes recent breakthroughs in GPCR crystallography and how this new information changed the way we approach GPCR drug-discovery.

2. Rask-Andersen M, Masuram S, Schioth HB: The druggable genome: Evaluation of drug targets in clinical trials suggests major shifts in molecular class and indication. Annu Rev Pharmacol Toxicol 2014, 54:9-26.

3. Wise A, Gearing K, Rees S: Target validation of G-protein coupled receptors. Drug Discov Today 2002, 7:235-246. 
4. Overington JP, Al-Lazikani B, Hopkins AL: How many drug targets are there? Nat Rev Drug Discov 2006, 5:993-996.

5. Heifetz A, Schertler GF, Seifert R, Tate CG, Sexton PM, Gurevich VV, Fourmy D, Cherezov V, Marshall FH, Storer RI, et al.: GPCR structure, function, drug discovery and crystallography: report from Academia-Industry International Conference (UK Royal Society) Chicheley Hall, 1-2 September 2014. Naunyn Schmiedebergs Arch Pharmacol 2015, 388:883-903.

6. Dohlman HG: Thematic Minireview Series: New Directions in G Protein-coupled Receptor Pharmacology. J Biol Chem 2015, 290:19469-19470.

7. Jazayeri A, Dias JM, Marshall FH: From G Protein-coupled Receptor Structure Resolution to Rational Drug Design. J Biol Chem 2015, 290:19489-19495.

-. Important review that outline some of the approaches used in GPCR structure-based drug design and describes technical solutions that have accelerated GPCR crystallography as well as some of the salient findings from structures that are relevant to drug discovery.

8. Bermudez M, Wolber G: Structure versus function-The impact of computational methods on the discovery of specific GPCR-ligands. Bioorg Med Chem 2015, 23:3907-3912.

9. Topiol S, Sabio M: The role of experimental and computational structural approaches in 7TM drug discovery. Expert Opin Drug Discov 2015, 10:1071-1084.

10. Ciancetta A, Sabbadin D, Federico S, Spalluto G, Moro S: Advances in Computational Techniques to Study GPCR-Ligand Recognition. Trends Pharmacol Sci 2015, 36:878-890.

11. Ciociola AA, Cohen LB, Kulkarni P, the FDARMCotACoG: How Drugs are Developed and Approved by the FDA: Current Process and Future Directions. Am J Gastroenterol 2014, 109:620-623.

12. Schmidt T, Bergner A, Schwede T: Modelling three-dimensional protein structures for applications in drug design. Drug discov. today 2014, 19:890-897.

13. Storer RI, Brennan PE, Brown AD, Bungay PJ, Conlon KM, Corbett MS, DePianta RP, Fish PV, Heifetz A, Ho DK, et al.: Multiparameter optimization in CNS drug discovery: design of pyrimido[4,5d]azepines as potent 5-hydroxytryptamine $2 \mathrm{C}(5-\mathrm{HT}(2) \mathrm{C})$ receptor agonists with exquisite functional selectivity over 5-HT(2)A and 5-HT(2)B receptors. J Med Chem 2014, 57:5258-5269.

14. Tautermann CS, Seeliger D, Kriegl JM: What can we learn from molecular dynamics simulations for GPCR drug design? Computational and Structural Biotechnology Journal 2015, 13:111-121.

- $\quad$ Important article that describes the application of molecular dynamics simulations in GPCR drug discovery programs, their scope and limitations. The application of MD to GPCR SBDD is exemplified by two selected case studies: discovery of small molecule antagonists of the human CC chemokine receptor 3 and a detailed investigation of the interplay between receptor dynamics and solvation for the binding of small molecules to the human muscarinic acetylcholine receptor 3 .

15. Tautermann CS: GPCR structures in drug design, emerging opportunities with new structures. Bioorg Med Chem Lett 2014, 24:4073-4079.

16. Heifetz A, Storer RI, McMurray G, James T, Morao I, Aldeghi M, Bodkin MJ, Biggin PC: Application of an Integrated GPCR SAR-Modelling Platform to Explain the Activation Selectivity of Human 5-HT2C over 5-HT2B. ACS Chem Biol 2016.

17. Jacobson KA: New paradigms in GPCR drug discovery. Biochem Pharmacol 2015, 98:541-555.

18. Heifetz A, Barker O, Morris GB, Law RJ, Slack M, Biggin PC: Toward an understanding of agonist binding to human Orexin-1 and Orexin-2 receptors with G-protein-coupled receptor modeling and site directed mutagenesis. Biochemistry 2013, 52:8246-8260.

19. Fenalti G, Giguere PM, Katritch V, Huang XP, Thompson AA, Cherezov V, Roth BL, Stevens RC: Molecular control of delta-opioid receptor signalling. Nature 2014, 506:191-196.

-• Highly important research that describes the solution of the high-resolution X-ray crystal structure of the human $\delta$-opioid receptor, revealing the presence and fundamental role of a sodium ion and water molecule network in mediating allosteric control of receptor functional selectivity and constitutive activity.

20. Liu W, Chun E, Thompson AA, Chubukov P, Xu F, Katritch V, Han GW, Roth CB, Heitman LH, AP IJ, et al.: Structural basis for allosteric regulation of GPCRs by sodium ions. Science 2012, 337:232-236.

21. Nygaard R, Frimurer TM, Holst B, Rosenkilde MM, Schwartz TW: Ligand binding and micro-switches in 7TM receptor structures. Trends Pharmacol Sci 2009, 30:249-259. 
22. Ross GA, Morris GM, Biggin PC: Rapid and accurate prediction and scoring of water molecules in protein binding sites. PLoS One 2012, 7:e32036.

23. Truchon JF, Pettitt BM, Labute P: A Cavity Corrected 3D-RISM Functional for Accurate Solvation Free Energies. J Chem Theory Comput 2014, 10:934-941.

24. Bortolato A, Tehan BG, Bodnarchuk MS, Essex JW, Mason JS: Water network perturbation in ligand binding: adenosine A(2A) antagonists as a case study. J Chem Inf Model 2013, 53:1700-1713.

25. Swinney DC: The role of binding kinetics in therapeutically useful drug action. Curr. Opin. Drug Discov. Devel. 2009, 12:31-39.

26. Keserü GM, Swinney DC (Ed): Thermodynamics and Kinetics of Drug Binding: Wiley-VCH, Verlag GmbH \& Co. KGaA, Heidelberg, Germany. ; 2015.

27. Cusack KP, Wang Y, Hoemann MZ, Marjanovic J, Heym RG, Vasudevan A: Design strategies to address kinetics of drug binding and residence time. Bioorg. Med. Chem. Lett. 2015, 25:2019-2027.

28. Guo D, Hillger JM, ljzerman AP, Heitman LH: Drug-target residence time-a case for G Protein-Coupled Receptors. Med. Res. Rev. 2014, 34:856-892.

- $\quad$ This article outlines and exemplifies the importance of drug-target residence times of GPCR drugs The authors propose that drug-target RT should be taken into account as an additional parameter in the lead selection and optimization process. This should ultimately lead to an increased number of candidate drugs moving to the preclinical development phase and onto the market. This review contains examples of the kinetic behavior of GPCR ligands with improved in vivo efficacy and summarizes methods for assessing drug-target RT.

29. Guo D, Mulder-Krieger T, ljzerman AP, Heitman LH: Functional efficacy of adenosine A2A receptor agonists is positively correlated to their receptor residence time. Br. J. Pharm. 2012, 166:18461859.

30. Vauquelin G: On the 'micro'-pharmacodynamic and pharmacokinetic mechanisms that contribute to long-lasting drug action. Expert Opin Drug Discov 2015:1-14.

31. Copeland RA: The dynamics of drug-target interactions: drug-target residence time and its impact on efficacy and safety. Expert Opin Drug Discov 2010, 5:305-310.

32. Hoffmann C, Castro M, Rinken A, Leurs R, Hill SJ, Vischer HF: Ligand Residence Time at G-proteinCoupled Receptors-Why We Should Take Our Time To Study It. Mol Pharmacol 2015, 88:552-560.

33. Frembgen-Kesner $\mathrm{T}$, Elcock $\mathrm{AH}$ : Computational sampling of a cryptic drug binding site in a protein receptor: Explicit solvent molecular dynamics and inhibitor docking to p38 MAP kinase. J. Mol. Biol. 2006, 359:202-214.

34. Lukman S, Verma C, Fuentes G: Exploiting protein intrinsic flexibility in drug design. In Protein Conformational Dynamics. Edited by Han K-I, Zhang X, Yang M-j: Springer International Publishing; 2014:245-269. Advances in Experimental Medicine and Biology, vol 805.]

35. Labute P: LowModeMD--implicit low-mode velocity filtering applied to conformational search of macrocycles and protein loops. J Chem Inf Model 2010, 50:792-800.

36. Tye H, Mueller SG, Prestle J, Scheuerer S, Schindler M, Nosse B, Prevost N, Brown CJ, Heifetz A, Moeller $\mathrm{C}$, et al.: Novel 6,7,8,9-tetrahydro-5H-1,4,7,10a-tetraaza-cyclohepta[f]indene analogues as potent and selective 5-HT(2C) agonists for the treatment of metabolic disorders. Bioorg Med Chem Lett 2011, 21:34-37.

37. Han M, Lou J, Nakanishi K, Sakmar TP, Smith SO: Partial agonist activity of 11-cis-retinal in rhodopsin mutants. J. Biol. Chem. 1997, 272:23081-23085.

38. Holst B, Nygaard R, Valentin-Hansen L, Bach A, Engelstoft MS, Petersen PS, Frimurer TM, Schwartz TW: A conserved aromatic lock for the tryptophan rotameric switch in TM-VI of seventransmembrane receptors. J. Biol. Chem. 2010, 285:3973-3985.

39. Schwartz TW, Frimurer TM, Holst B, Rosenkilde MM, Elling CE: Molecular mechanism of 7tm receptor activation-a global toggle switch model. Ann. Rev. Pharmacol. Toxicol. 2006, 46:481-519.

40. Tehan BG, Bortolato A, Blaney FE, Weir MP, Mason JS: Unifying family A GPCR theories of activation. Pharm. Res. 2014, 143:51-60.

41. Heifetz AB, O.; Verquin, G.; Wimmer, N.; Meutermans, W.; Pal, S.; Law, R. J.; Whittaker, M. : Fighting obesity with a sugar-based library: Discovery of novel $\mathrm{MCH}-1 \mathrm{R}$ antagonists by a new computational-VAST approach for exploration of GPCR binding sites. J. Chem. Inf. Model. 2013, 53:1084-1099.

42. Boss C, Roch C: Recent trends in orexin research--2010 to 2015. Bioorg Med Chem Lett 2015, 25:28752887.

43. Yin J, Babaoglu K, Brautigam CA, Clark L, Shao Z, Scheuermann TH, Harrell CM, Gotter AL, Roecker AJ, Winrow CJ, et al.: Structure and ligand-binding mechanism of the human OX and OX orexin receptors. Nat Struct Mol Biol 2016. 
44. Heifetz A, Morris GB, Biggin PC, Barker O, Fryatt T, Bentley J, Hallett D, Manikowski D, Pal S, Reifegerste $R$, et al.: Study of human Orexin-1 and -2 -G-protein-coupled receptors with novel and published antagonists by modeling, molecular dynamics simulations, and site-directed mutagenesis. Biochemistry 2012, 51:3178-3197.

45. Heifetz A, Chudyk El, Gleave L, Aldeghi M, Cherezov V, Fedorov DG, Biggin PC, Bodkin MJ: The Fragment Molecular Orbital Method Reveals New Insight into the Chemical Nature of GPCR-Ligand Interactions. J Chem Inf Model 2016, 56:159-172.

- $\quad$ Article that describes the application of the QM method FMO in the exploration of GPCR-ligand interactions.

46. Heifetz A, Aldeghi M, Chudyk E, Fedorov DG, Bodkin M, Biggin PC: Using the Fragment Molecular Orbital Method to Investigate Agonist-Orexin 2 Receptor Interactions Biochemical Society Transactions 2016.

47. Yin J, Mobarec JC, Kolb P, Rosenbaum DM: Crystal structure of the human OX2 orexin receptor bound to the insomnia drug suvorexant. Nature 2015, 519:247-250. 